\title{
MODELS OF TORSORS AND THE FUNDAMENTAL GROUP SCHEME
}

\author{
MARCO ANTEI AND MICHEL EMSALEM
}

\begin{abstract}
Given a relative faithfully flat pointed scheme over the spectrum of a discrete valuation ring $X \rightarrow S$, this paper is motivated by the study of the natural morphism from the fundamental group scheme of the generic fiber $X_{\eta}$ to the generic fiber of the fundamental group scheme of $X$. Given a torsor $T \rightarrow X_{\eta}$ under an affine group scheme $G$ over the generic fiber of $X$, we address the question of finding a model of this torsor over $X$, focusing in particular on the case where $G$ is finite. We provide several answers to this question, showing for instance that, when $X$ is integral and regular of relative dimension 1, such a model exists on some model $X^{\prime}$ of $X_{\eta}$ obtained by performing a finite number of Néron blowups along a closed subset of the special fiber of $X$. Furthermore, we show that when $G$ is étale, then we can find a model of $T \rightarrow X_{\eta}$ under the action of some smooth group scheme. In the first part of the paper, we show that the relative fundamental group scheme of $X$ has an interpretation as the Tannaka Galois group of a Tannakian category constructed starting from the universal torsor.
\end{abstract}

\section{$\S 1$. Introduction}

\subsection{Aim and scope}

Let $S$ be a Dedekind scheme of dimension 1 , and let $\eta=\operatorname{Spec}(K)$ be its generic point; let $X$ be a scheme, let $f: X \rightarrow S$ be a faithfully flat morphism of finite type, and let $f_{\eta}: X_{\eta} \rightarrow \eta$ be its generic fiber. Assume that we are given a finite $K$-group scheme $G$ and a $G$-torsor $Y \rightarrow X_{\eta}$. So far, the problem of extending the $G$-torsor $Y \rightarrow X_{\eta}$ has consisted in finding a finite and flat $S$-group scheme $G^{\prime}$ whose generic fiber is isomorphic to $G$, and a $G^{\prime}$-torsor $T \rightarrow X$ whose generic fiber is isomorphic to $Y \rightarrow X_{\eta}$ as a $G$-torsor. Some solutions to this problem, from Grothendieck's first ideas until the present, are known in some particular relevant cases that we

Received April 5, 2016. Revised November 17, 2016. Accepted November 17, 2016.

2010 Mathematics subject classification. Primary 14L30, 14L15; Secondary 11G99.

Marco Antei remercie le projet TOFIGROU (ANR-13-PDOC-0015-01). Michel Emsalem a reçu le soutien du Labex CEMPI (ANR-11-LABX-01).

(C) 2016 by The Editorial Board of the Nagoya Mathematical Journal 
briefly recall. Grothendieck proved that, possibly after extending scalars, the problem has a solution when $G$ is a constant finite group, and $S$ is the spectrum of a complete discrete valuation ring with algebraically closed residue field of positive characteristic $p$, with $X$ proper and smooth over $S$ with geometrically connected fibers and $p \nmid|G|$ (see [11, Exposé X]). When $S$ is the spectrum of a discrete valuation ring of residue characteristic $p$, and $X$ is a proper and smooth curve over $S$, then Raynaud suggested a solution, possibly after extending scalars, for $|G|=p$ (see [22, Section 3]). When $S$ is the spectrum of a discrete valuation $\operatorname{ring} R$ of mixed characteristic $(0, p)$, Tossici provided a solution, possibly after extending scalars, for $G$ commutative, when $X$ is a regular scheme, faithfully flat over $S$, with some extra assumptions on $X$ and $Y$ (see [24, Corollary 4.2.8]). Finally, in [2, Sections 3.2 and 3.3], the first author provided a solution for $G$ commutative, when $S$ is a connected Dedekind scheme and $f: X \rightarrow S$ is a smooth morphism satisfying additional assumptions (in this last case it is not needed to extend scalars). In [3], the case where $G$ is solvable is treated. However, a general solution does not exist. Moreover, we know that it can even happen that $G$ does not admit a finite and flat model. (See [17, Appendix B, Proposition B.2] for the positive equal characteristic case or [20, Section 3.4] for the mixed characteristic case.) What is always true is that $G$ admits at least an affine, quasi-finite (then, of finite type, according to our conventions, see Section 1.2), flat $R$-group scheme model. Indeed, $G$ is isomorphic to a closed subgroup scheme of some $\mathrm{GL}_{n, K}$ (see [25, Section 3.4]); then, it is sufficient to consider its schematic closure in $\mathrm{GL}_{n, S}$. In this paper, we explain how to solve the problem of extending any $G$-torsor when $G$ is any algebraic group scheme over $K$ up to a modification of $X$. We obtain, for instance, the following theorem.

THEOREM 3.9. Let $S$ be the spectrum of a Henselian discrete valuation ring $R$ with function field $K$ and with algebraically closed residue field. Let $X \rightarrow S$ be a smooth and surjective morphism with $X$ a connected scheme. Let $G$ be an affine $K$-group scheme of finite type, and let $f: Y \rightarrow X_{\eta}$ be a $G$-torsor; then, there exists, possibly after extension of scalars, a connected scheme $X^{\prime}$ smooth and surjective over $S$, a model map $\lambda: X^{\prime} \rightarrow X$ and a $G_{0}$-torsor $f^{\prime}: Y_{0} \rightarrow X^{\prime}$ extending the given $G$-torsor $Y$ for some affine finite type and flat $S$-group scheme $G_{0}$. If, moreover, $G$ is finite, then $G_{0}$ is quasifinite, and there exists an open subscheme $X_{1} \subseteq X$ such that $X \backslash X_{1}$ has codimension $\geqslant 3$ in $X$, and such that $X^{\prime}$ can be obtained from $X_{1}$ after a finite number of Néron blowups. 
When $X$ is a relative curve, this modification is represented by a finite number of Néron blowups of $X$ in a closed subscheme of the special fiber of $X$. For more precise statements, we refer the reader to Section 3 . The most interesting case is certainly the case where $G$ is finite. If we were able to prove that every finite and pointed torsor over $X_{\eta}$ admits a model over $X$, then the natural morphism $\varphi: \pi\left(X_{\eta}, x_{\eta}\right) \rightarrow \pi^{\mathrm{qf}}(X, x)_{\eta}$ (which is always faithfully flat, see [4, Section 7]) between the fundamental group scheme of $X_{\eta}$ and the generic fiber of the quasi-finite fundamental group scheme of $X$ would be an isomorphism. It is known that $\varphi$ becomes an isomorphism when we restrict to the abelianized fundamental group scheme (cf. [2]). Here, we find a partial answer, extending all finite torsors, but instead of providing a model over $X$, we provide a model over some $X^{\prime}$ obtained by slightly modifying $X$, as explained. Furthermore, when $G$ is finite étale, then we can iterate our techniques (we keep on Néron blowing up $X$, if necessary) in order to obtain a model for the given torsor under the action of a smooth group scheme (cf. Corollary 3.10); this is sharp in the sense that several examples of étale torsors whose models over $X$ are never smooth are known. In order to approach the question from a different point of view, it would be of great interest to have a Tannakian description for $\pi^{\mathrm{qf}}(X, x), \pi(X, x)$ and their universal torsors $\widehat{X}^{\mathrm{qf}} \rightarrow X$ and $\widehat{X} \rightarrow X$. In [15], Mehta and Subramanian provided a first construction which works only for schemes defined over some non-Noetherian Prüfer rings whose function field is algebraically closed. In Section 2, we give a different Tannakian description, simply choosing the category of vector bundles on $X$ trivialized by the universal torsor, whose existence is now known. An intrinsic description, independent from the existence of the universal torsor, would be strongly appreciated.

\subsection{Notations and conventions}

Let $S$ be any scheme, let $X$ be an $S$-scheme, let $G$ be a (faithfully) flat, affine $^{1} S$-group scheme, and let $Y$ be an $S$-scheme endowed with a right action $\sigma: Y \times G \rightarrow Y$. An $S$-morphism $p: Y \rightarrow X$ is said to be a $G$-torsor if it is faithfully flat, $G$-invariant, and the canonical morphism $\left(\sigma, p r_{Y}\right)$ : $Y \times G \rightarrow Y \times_{X} Y$ is an isomorphism. Let $H$ be a flat, affine $S$-group scheme, and let $q: Z \rightarrow X$ be an $H$-torsor; a morphism between two such torsors is a pair $(\beta, \alpha):(Z, H) \rightarrow(Y, G)$, where $\alpha: H \rightarrow G$ is an $S$-morphism of

\footnotetext{
${ }^{1}$ We do not need $G$ to be affine in order to define a $G$-torsor, but it is the only type we encounter in this paper.
} 
group schemes, and $\beta: Z \rightarrow Y$ is an $X$-morphism of schemes such that the following diagram commutes:

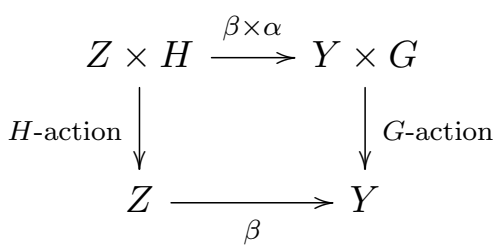

(thus $Y$ is isomorphic to the contracted product $Z \times{ }^{H} G$ through $\alpha$, cf. [6, III, Section 4, 3.2]). In this case, we say that $Z$ precedes $Y$.

When $S$ is irreducible, $\eta$ will denote its generic point and $K$ its function field $k(\eta)$. Any $S$-scheme whose generic fiber is isomorphic to some $K$ scheme $T_{\eta}$ will be called a model of $T_{\eta}$. Any morphism that is generically an isomorphism will be called a model map.

Throughout the whole paper, a morphism of schemes $f: Y \rightarrow X$ will be said to be quasi-finite if it is of finite type, and for every point $x \in X$, the fiber $Y_{x}$ is a finite set. Let $S$ be any scheme, and let $G$ be an affine $S$-group scheme. Then, we say that $G$ is a finite (resp. quasi-finite) $S$-group scheme if the structural morphism $G \rightarrow S$ is finite (resp. quasi-finite).

Let $R$ be a commutative ring with unity, and let $G$ be an affine and flat $R$ group scheme; we denote by $\operatorname{Rep}_{R, t f}(G)$ the category of finitely generated $R$-linear representations of $G$, and by $\operatorname{Rep}_{R, t f}^{0}(G)$ the full subcategory of $\operatorname{Rep}_{R, t f}(G)$ whose objects are free (as $R$-modules).

\section{§2. A Tannakian construction}

Throughout this section, $R$ will always be a discrete valuation ring, with uniformizing element $\pi$, with field of fractions $K:=\operatorname{Frac}(R)$ and residue field $k:=R / \pi R$. The generic and special points of $\operatorname{Spec}(R)$ will often be denoted by $\eta$ and $s$, respectively.

Lemma 2.1. Let $X$ be a Noetherian scheme over $R$, and let $T=\lim _{i \in I} T_{i}$ be a projective limit of $X$-schemes $f_{i}: T_{i} \rightarrow X$ affine over $X$. We assume that for all $i \in I, H^{0}\left(T_{i}, \mathcal{O}_{T_{i}}\right)=R$. Then, $H^{0}\left(T, \mathcal{O}_{T}\right)=R$.

Proof. This follows from [13, III, Proposition 2.9].

LEMMA 2.2. Let $j: T \rightarrow \operatorname{Spec}(R)$ be a quasi-compact and quasiseparated faithfully flat morphism, and let us assume that the generic fiber $T_{\eta}$ of $T$ is such that $H^{0}\left(T_{\eta}, \mathcal{O}_{T_{\eta}}\right)=K$. Then, $H^{0}\left(T, \mathcal{O}_{T}\right)=R$. 
Proof. We first observe that either $H^{0}\left(T, \mathcal{O}_{T}\right)=R$ or $H^{0}\left(T, \mathcal{O}_{T}\right)=K$. Indeed,

$$
R \subset H^{0}\left(T, \mathcal{O}_{T}\right) \subset H^{0}\left(T, \mathcal{O}_{T}\right) \otimes_{R} K \simeq H^{0}\left(T_{\eta}, \mathcal{O}_{T_{\eta}}\right)=K,
$$

the last isomorphism being a consequence of [23, Lemma 29.5.2], and it is not difficult to check that an $R$-algebra containing $R$ and contained in $K$ is either $R$ or $K$. However, if $H^{0}\left(T, \mathcal{O}_{T}\right)=K, f$ would not be surjective because of the canonical factorization of $f: T \rightarrow \operatorname{Spec}(R)$ into $T \rightarrow \operatorname{Spec}\left(\mathcal{O}_{T}(T)\right) \rightarrow$ $\operatorname{Spec}(R)$.

We apply these remarks to the theory of the (quasi-finite) fundamental group scheme and its universal torsor which we briefly recall.

Definition 2.3. Let $X \rightarrow S$ be a morphism of schemes endowed with a section $x \in X(S)$. We say that $X$ has a fundamental group scheme (resp. a quasi-finite fundamental group scheme) if there exist an $S$-group scheme $\pi(X, x)$ (resp. $\left.\pi^{\mathrm{qf}}(X, x)\right)$ and a pointed $\pi(X, x)$-torsor $\widehat{X}$ (resp. $\pi^{\mathrm{qf}}(X, x)$ torsor $\widehat{X}^{\mathrm{qf}}$ ) such that for any finite (resp. quasi-finite) torsor $Y \rightarrow X$ over $X$, pointed over $x$, there is a unique morphism of pointed torsors $\widehat{X} \rightarrow Y$ (resp. $\left.\widehat{X}^{\mathrm{qf}} \rightarrow Y\right)$.

In [4, Sections 4, 5.1 and 5.2], we proved the following existence theorems.

THEOREM 2.4. Let $S$ be a Dedekind scheme, let $X \rightarrow S$ be a faithfully flat morphism locally of finite type, and let $x \in X(S)$ be a section. Let us moreover assume that one of the following assumptions is satisfied:

(1) for every $s \in S$, the fiber $X_{s}$ is reduced;

(2) for every $z \in X \backslash X_{\eta}$, the local ring $\mathcal{O}_{X, z}$ is integrally closed.

Then, $X$ has a fundamental group scheme.

TheOREM 2.5. Let $S$ be a Dedekind scheme, let $X \rightarrow S$ be a faithfully flat morphism locally of finite type, and let $x \in X(S)$ be a section. Let us moreover assume that $X$ is normal, and that for each $s \in S$, the fiber $X_{s}$ is normal and integral. (Note that these conditions imply that $X$ is integral.) Then, $X$ has a quasi-finite fundamental group scheme.

Notation 2.6. In order to simplify the exposition, from now on we only consider the case where $X$ satisfies the assumptions of Theorem 2.5. However, the statements proved for the universal $\pi^{\mathrm{qf}}(X, x)$-torsor will also hold for the universal $\pi(X, x)$-torsor, and the proofs are exactly the same. 
Definition 2.7. We say that a quasi-finite $G$-torsor over $X$ pointed over $x$ is quasi-Galois if the canonical morphism $\rho: \pi(X, x)^{\mathrm{qf}} \rightarrow G$ is schematically dominant (that is, $R[G] \rightarrow R\left[\pi(X, x)^{\mathrm{qf}}\right]$ is injective). It is furthermore called Galois, if $\rho: \pi(X, x)^{\mathrm{qf}} \rightarrow G$ is faithfully flat.

LEMMA 2.8. Consider a faithfully flat morphism $X \rightarrow \operatorname{Spec}(R)$ of finite type. Let us assume that $H^{0}\left(X, \mathcal{O}_{X}\right)=R$. Let $G$ be a quasi-finite and flat group scheme, and let $T \rightarrow X$ be a quasi-Galois $G$-torsor pointed over $x$. Then, $H^{0}\left(T, \mathcal{O}_{T}\right)=R$.

Proof. It is sufficient to notice that the generic fiber $T_{\eta}$ of $T$ is Galois over $X_{\eta}$ (cf. [4, Section 7, Lemma 7.6]). Hence, $H^{0}\left(T_{\eta}, \mathcal{O}_{T_{\eta}}\right)=K$ (cf. [18, Chapter 2, Proposition 3]). The conclusion follows by Lemma 2.2.

Corollary 2.9. Consider a faithfully flat morphism $X \rightarrow \operatorname{Spec}(R)$ of finite type. Let us assume that $H^{0}\left(X, \mathcal{O}_{X}\right)=R$. Then, $H^{0}\left(\widehat{X}^{\mathrm{qf}}, \mathcal{O}_{\widehat{X}^{\mathrm{qf}}}\right)=R$.

Proof. First, we observe that for any quasi-finite and flat $R$-group scheme $G$, any $G$-torsor over $X$ pointed over $x$ is preceded by a quasiGalois torsor: it is sufficient to factor the canonical morphism $\pi^{\mathrm{qf}}(X, x) \rightarrow G$ as $\pi^{\mathrm{qf}}(X, x) \rightarrow G^{\prime} \rightarrow G$, where $\pi^{\mathrm{qf}}(X, x) \rightarrow G^{\prime}$ is a schematically dominant morphism and $G^{\prime} \rightarrow G$ is a closed immersion. Then, the contracted product $\widehat{X}^{\mathrm{qf}} \times^{\pi^{\mathrm{qf}}(X, x)} G^{\prime}$ gives the desired quasi-Galois torsor. Hence, the universal torsor is isomorphic to a projective limit of quasi-Galois torsors, and the conclusion follows using Lemmas 2.8 and 2.1.

TheOREm 2.10. With the same assumptions as in Corollary 2.9, the

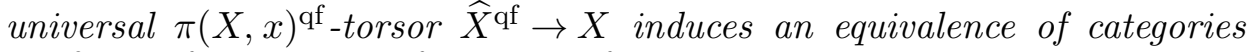
$\operatorname{Rep}_{R, t f}^{0}\left(\pi^{\mathrm{qf}}(X, x)\right) \rightarrow \mathcal{T}^{\mathrm{qf}}$, where $\mathcal{T}^{\mathrm{qf}}$ denotes the category of vector bundles on $X$ trivialized by $\widehat{X}^{\mathrm{qf}} \rightarrow X$.

Let $\theta: X \rightarrow \operatorname{Spec}(R)$ denote the structural morphism, and let $j: T \rightarrow X$ be any $G$-torsor for some affine and flat $R$-group scheme $G$, then we denote by $G-\operatorname{Vec} t_{T}$ the category of $G$-sheaves over $T$ whose objects are locally free as $\mathcal{O}_{T}$-modules. It is known that the functor $j^{*}: \operatorname{Vec} t_{X} \rightarrow G-\operatorname{Vec} t_{T}$ is an equivalence of categories, and we denote by $\rho: G-\operatorname{Vec} t_{T} \rightarrow \operatorname{Vec} t_{X}$ a quasi-inverse. As usual, we naturally associate to $j: T \rightarrow X$ the functor $F_{T}: \operatorname{Rep}_{R, t f}^{0} G \rightarrow \mathcal{T}$, where $\mathcal{T}$ denotes the category of vector bundles on $X$ trivialized by $T \rightarrow X$, given by $\rho \circ\left(j^{*} \circ \theta^{*}\right)$. Thus, Theorem 2.10 is a consequence of the following more general statement, which somehow generalizes an analog result for torsors over fields (cf. [18, Chapter II, Proposition 3]). 
Lemma 2.11. Consider a faithfully flat morphism $X \rightarrow \operatorname{Spec}(R)$ of finite type. Let $G \rightarrow \operatorname{Spec}(R)$ be a flat affine group scheme, and let $j: T \rightarrow X$ be a $G$-torsor such that $H^{0}\left(T, \mathcal{O}_{T}\right)=R$. The functor $F_{T}: \operatorname{Rep}_{R, t f}^{0} G \rightarrow \mathcal{T}$ is an equivalence of categories.

Proof. First, we observe that the functor $F_{T}$ is fully faithful if and only if $\left(j^{*} \circ \theta^{*}\right)$ is fully faithful. Let $V_{1}, V_{2}$ be two objects of $\operatorname{Rep}_{R, t f}^{0} G$. Then, $\operatorname{Hom}_{\left(\operatorname{Rep}_{R, t f}^{0} G\right)}\left(V_{1}, V_{2}\right)=\left(V_{1}^{\vee} \otimes_{R} V_{2}\right)^{G}$. Analogously, if $\mathcal{F}_{1}, \mathcal{F}_{2}$ are two objects of $G-\operatorname{Vec} t_{T}$, then

$$
\operatorname{Hom}_{G-\operatorname{Vec}_{T}}\left(\mathcal{F}_{1}, \mathcal{F}_{2}\right)=H^{0}\left(T, \mathcal{F}_{1}^{\vee} \otimes \mathcal{O}_{T} \mathcal{F}_{2}\right)^{G}
$$

Thus, $F_{T}$ is fully faithful if and only if, for any object $W$ of $\operatorname{Rep}_{R, t f}^{0} G$, the natural map

$$
W^{G} \rightarrow H^{0}\left(T, j^{*} \theta^{*}(W)\right)^{G}
$$

is an isomorphism. We have the following sequence of isomorphisms (by means of the projection formula):

$$
\begin{aligned}
& H^{0}\left(T, j^{*} \theta^{*}(W)\right) \\
& \quad \simeq H^{0}\left(\operatorname{Spec}(R),(\theta \circ j)_{*}(\theta \circ j)^{*}(W)\right) \simeq H^{0}\left(\operatorname{Spec}(R),(\theta \circ j)_{*} \mathcal{O}_{T} \otimes_{R} W\right) \\
& \simeq H^{0}\left(\operatorname{Spec}(R),(\theta \circ j)_{*} \mathcal{O}_{T}\right) \otimes_{R} W \simeq H^{0}\left(T, \mathcal{O}_{T}\right) \otimes_{R} W
\end{aligned}
$$

as representations of $G$. Then, $\left(H^{0}\left(T, j^{*} \theta^{*}(W)\right)\right)^{G} \simeq\left(H^{0}\left(T, \mathcal{O}_{T}\right) \otimes_{R} W\right)^{G}$ $=W^{G}$, since we assumed $H^{0}\left(T, \mathcal{O}_{T}\right)=R$, whence we have the desired isomorphism $(\dagger)$. In order to prove the essential surjectivity, we argue as follows. Let us take a vector bundle $E$ on $X$ trivialized by $j: T \rightarrow X$. That implies the existence of a finitely generated free $R$-module $M$ such that $\mathcal{E}:=j^{*} E \simeq(\theta \circ j)^{*} M$. Again applying the projection formula, we obtain

$$
(\theta \circ j)_{*}(\theta \circ j)^{*} M=(\theta \circ j)_{*} \mathcal{O}_{T} \otimes_{R} M=M .
$$

It follows that

$$
\mathcal{E} \simeq(\theta \circ j)^{*}(\theta \circ j)_{*}(\theta \circ j)^{*} M \simeq(\theta \circ j)^{*}(\theta \circ j)_{*} \mathcal{E} \simeq(\theta \circ j)^{*} H^{0}(T, \mathcal{E})
$$

We now observe that the previous isomorphism $(\theta \circ j)^{*}(\theta \circ j)_{*} \mathcal{E} \rightarrow \mathcal{E}$ is $G$ equivariant, and thus $F_{T}\left(H^{0}(T, \mathcal{E})\right) \simeq E$. 
REMARK 2.12. Lemma 2.11 can be generalized further as follows. Let $R$ be any commutative and unitary ring, and let $q: T \rightarrow \operatorname{Spec}(R)$ be a morphism of scheme such that $H^{0}\left(T, \mathcal{O}_{T}\right)=R$. Let moreover $G$ be any flat and affine $R$-group scheme, acting on $T$, and let $\mathcal{F}$ be any $G$-sheaf, trivial as an $\mathcal{O}_{T}$-module. Then, $H^{0}(T, \mathcal{F})$ is an $R$-linear representation of $G$, and $\mathcal{F} \simeq H^{0}(T, \mathcal{F}) \otimes_{R} \mathcal{O}_{T}$ as $G$-sheaves.

REMARK 2.13. With notations as in Lemma 2.11, the Tannakian category $\mathcal{C}$ over $R$ (cf. [7] for a modern and detailed overview) associated to $\mathcal{T}$ is the category of those $\mathcal{O}_{X}$-modules whose pullback over $T$ is isomorphic, as $\mathcal{O}_{T}$-module, to a finite direct sum of $\mathcal{O}_{T}$ and $\mathcal{O}_{T} / \pi^{n}$, where $\pi$ denotes a uniformizer of $R$ and $n$ is a natural integer. In this way, $\mathcal{T}$ would coincide with the full subcategory $\mathcal{C}^{0}$ of $\mathcal{C}$ of rigid objects of $\mathcal{C}$; that is, objects isomorphic to their double dual. It would be very interesting and useful to have an inner description of the objects of $\mathcal{T}$ (or equivalently of $\mathcal{C}$ ) independent from the universal torsor.

\section{$\S 3 . \quad$ Existence of a model}

\subsection{Quotients and Néron blowups}

In this section, we recall some results ensuring the existence of quotients of schemes under the action of some group schemes, under certain assumptions. These results are essentially contained in [11, Exposé V, théorème 7.1] and $[21$, théorème $1,(\mathrm{iv})]$ for the finite case, and $[21$, théorème $1,(\mathrm{v})]$ and $[1$, théorème 7 , appendice 1] for the quasi-finite case. The fact that quotients (under the action of finite type group schemes) commute with base change is ensured by [11, Exposé IV, 3.4.3.1].

TheOrem 3.1. Let $T$ be a locally Noetherian scheme, let $Z$ be a $T$ scheme locally of finite type, and let $H$ be a flat $T$-group scheme acting on $Z$ in such a way that $Z \times_{T} H \rightarrow Z \times_{T} Z$ is a closed immersion. Then, if one of the following conditions is verified:

(1) $H \rightarrow T$ is finite and $Z \rightarrow T$ is quasi-projective,

(2) $H \rightarrow T$ is quasi-finite and $Z \rightarrow T$ is quasi-finite,

the sheaf $(Z / H)_{f p q c}$ is represented by a scheme $Z / H$. Moreover, the canonical morphism $Z \rightarrow Z / H$ is faithfully flat, and the natural morphism $Z \times{ }_{T} H \rightarrow Z \times{ }_{Z / H} Z$ is an isomorphism.

TheOrem 3.2. Let $T$ be any locally Noetherian scheme, let $Z$ be a $T$ scheme locally of finite type, and let $H$ be a flat $T$-group scheme acting on $Z$ 
such that the natural morphism $Z \times_{T} H \rightarrow Z \times_{T} Z$ is a closed immersion. Then, there exists a largest open $U$ of $Z$ for which the sheaf $(U / H)_{\text {fpqc }}$ is represented by a scheme $U / H$. Moreover, $U$ is dense in $Z$ and contains the points of $Z$ of codimension $\leqslant 1$. Furthermore, the canonical morphisms $U \rightarrow U / H$ is faithfully flat.

Proof. It was first stated in [21, Théorème 1(i)], and a proof is contained in [1, Proposition 3.3.1]. The last assertion is just [21, Section 4, Proposition 2].

The conclusion is that, in both cases (Theorems 3.1 and 3.2), $Z \rightarrow Z / H$ and $U \rightarrow U / H$ are $H$-torsors.

Corollary 3.3. Let $S$ be a Dedekind scheme with function field $K$, and let $X \rightarrow S$ be a faithfully flat morphism of finite type. Moreover, let $G^{\prime}$ be an affine and flat $S$-group scheme, let $Z$ be a faithfully flat $S$-scheme of finite type provided with a right $G^{\prime}$-action $\sigma: Z \times{ }_{S} G^{\prime} \rightarrow Z$, and let $g: Z \rightarrow X$ be a $G^{\prime}$-invariant (that is $g \circ \sigma=g \circ p r_{Z}$ ) S-morphism such that the natural morphism $Z \times{ }_{S} G^{\prime} \rightarrow Z \times{ }_{X} Z$ is a closed immersion inducing a $G_{\eta}^{\prime}$-torsor structure on $Z_{\eta}$ over $X_{\eta}$. Let $U$ be the largest open of $Z$ as in Theorem 3.2 such that $U / G^{\prime}$ is a scheme; then, $X^{\prime}:=U / G^{\prime}$ is faithfully flat and of finite type over $S$, and the natural morphism $\lambda: X^{\prime} \rightarrow X$ is a model map. In particular, $U \rightarrow X^{\prime}$ is a $G^{\prime}$-torsor extending the $G_{\eta}^{\prime}$-torsor $Z_{\eta} \rightarrow X_{\eta}$.

Proof. By Theorem 3.2, $U$ contains the points of $Z$ of codimension $^{2} \leqslant 1$, so in particular it contains, for all closed points $s \in S$, the generic points of the irreducible components of $Z_{s}$. As $U$ is the largest open of $Z$ such that $U / G^{\prime}$ is a scheme, so in particular it contains $Z_{\eta}$. Thus, $U$ is surjective over $S$. Hence, $X^{\prime}$ is surjective over $S$ too, and it is $S$-flat and of finite type because $U$ has the same properties (inherited by $Z$ ). Thus, $X^{\prime} \rightarrow X$ gives rise to the desired model map. The last assertion is clear.

We now recall the definition of Néron blowup. Unless stated otherwise, from now until the end of Section 3.1, we only consider the following situation.

Notation 3.4. We denote by $S$ the spectrum of a discrete valuation $\operatorname{ring} R$ with uniformizing element $\pi$, and with fraction and residue field respectively denoted by $K$ and $k$. As usual, $\eta$ and $s$ denote the generic and special points

\footnotetext{
${ }^{2}$ The codimension of a point is defined as the codimension of its closure.
} 
of $S$, respectively. Finally, we denote by $X$ a faithfully flat and separated $S$-scheme of finite type.

According to $[5$, Section 3.2 Proposition 1] or $[1$, II, 2.1.2(A)], the following statement holds.

Proposition 3.5. Let $S$ be the spectrum of a discrete valuation ring $R$ with uniformizing element $\pi$. Let $X$ be a faithfully flat $S$-scheme of finite type, let $C$ be a closed subscheme of the special fiber $X_{s}$ of $X$, and let $\mathcal{I}$ be the sheaf of ideals of $\mathcal{O}_{X}$ defining $C$. Let $X^{\prime} \rightarrow X$ be the blowup of $X$ at $C$, and let $u: X^{C} \rightarrow X$ denote its restriction to the open subscheme of $X^{\prime}$, where $\mathcal{I} \cdot \mathcal{O}_{X}$ is generated by $\pi$. Then:

(1) $X^{C}$ is a flat $S$-scheme of finite type, $u$ is an affine model map;

(2) for any flat $S$-scheme $Z$ and for any $S$-morphism $v: Z \rightarrow X$ such that $v_{k}$ factors through $C$, there exists a unique $S$-morphism $v^{\prime}: Z \rightarrow X^{C}$ such that $v=u \circ v^{\prime}$.

Definition 3.6. The morphism $X^{C} \rightarrow X$ (or simply $X^{C}$ ) as in Proposition 3.5 is called the Néron blowup of $X$ at $C$, and property (2) is often referred to as the universal property of the Néron blowup.

Now, we explain how to Néron blow-up torsors.

Proposition 3.7. Let $G$ be an affine, algebraic and flat $S$-group scheme, and let $H$ be a closed subgroup scheme of $G_{s}$. Let $Y$ be a $G$-torsor over $X$, and let $Z$ be an $H$-torsor over $X_{s}$, subtorsor of $Y_{s} \rightarrow X_{s}$. Then, there exist a faithfully flat $S$-scheme of finite type $X^{\prime}$, and a model map $\lambda: X^{\prime} \rightarrow X$ such that $Y^{Z} \rightarrow X^{\prime}$ is a $G^{H}$-torsor generically isomorphic to $Y_{\eta} \rightarrow X_{\eta}$. If, moreover, $G$ is quasi-finite, then $\lambda$ can be obtained from $X$ after a finite number of Néron blowups.

Proof. From the universal property of Néron blowups, we first obtain an action of $G^{H}$ on $Y^{Z}$. Indeed, $Y^{Z} \times G^{H} \rightarrow Y \times G \rightarrow Y$ (the last morphism is the action of $G$ on $Y$ ) specially factors through $Z$, whence we have a morphism $Y^{Z} \times G^{H} \rightarrow Y^{Z}$ that gives the desired action. Under this action, $Y^{Z} \rightarrow X$ is $G^{H}$-invariant; then, we have a natural morphism $Y^{Z} \times G^{H} \rightarrow$ $Y^{Z} \times_{X} Y^{Z}$. Moreover, $Y^{Z} \times_{X} Y^{Z} \rightarrow Y \times_{X} Y \simeq Y \times_{S} G$ specially factors through $Z \times H$; then, we obtain a morphism $Y^{Z} \times_{X} Y^{Z} \rightarrow Y^{Z} \times G^{H}$. As $Y^{Z} \times G^{H}$ is $S$-flat, the generic fiber $\left(Y^{Z} \times G^{H}\right)_{\eta}$ is schematically dominant in $Y^{Z} \times G^{H}$. As $Y^{Z} \times G^{H}$ is separated over $S$ and the composition morphism $Y^{Z} \times G^{H} \rightarrow Y^{Z} \times{ }_{X} Y^{Z} \rightarrow Y^{Z} \times G^{H}$ is generically the identity, 
according to [23, Lemma 28.7.10], it is the identity; hence, $Y^{Z} \times G^{H} \rightarrow$ $Y^{Z} \times_{X} Y^{Z}$ is a monomorphism and is proper according to [14, Proposition 3.3.16(e)], and is thus a closed immersion. Using Corollary 3.3, one gets the conclusion. If $G$ is quasi-finite, the open $U$ in Corollary 3.3 is the whole $Y^{Z}$. In this case, $\lambda: Y^{Z} / G^{H}=X^{\prime} \rightarrow X$ is affine and can be expressed as a finite number of Néron blowups (cf. [26, Theorem 1.4]).

\subsection{Construction of a model}

Given an algebraic $G$-torsor $Y \rightarrow X_{\eta}$, we do not know whether or not we can find a torsor over $X$ whose generic fiber is isomorphic to the given one. In Section 1.1, we recall the most important and recent results that partially solve this problem when $G$ is finite. Here, we suggest a new approach in a much wider context, including the case $G$ of finite type.

THEOREM 3.8. Let $S$ be the spectrum of a discrete valuation ring $R$ with function field $K$. Let $X \rightarrow S$ be a faithfully flat morphism of finite type, with $X$ a regular and integral scheme of absolute dimension 2 endowed with a section $x \in X(S)$. Let $G$ be a finite $K$-group scheme, and let $f: Y \rightarrow X_{\eta}$ be a G-torsor pointed in $y \in Y_{x_{\eta}}(K)$; then, there exist an integral scheme $X^{\prime}$ faithfully flat and of finite type over $S$, a model map $\lambda: X^{\prime} \rightarrow X$ and a $G_{0}$-torsor $f^{\prime}: Y_{0} \rightarrow X^{\prime}$ extending the given $G$-torsor $Y$ for some quasi-finite and flat $S$-group scheme $G_{0}$. Moreover, $Y_{0}$ comes with a $R$-point $y_{0} \in Y_{0}(R)$, whose restriction to $X_{\eta}$ is the given point $y$. Furthermore, $X^{\prime}$ can be obtained from $X$ after a finite number of Néron blowups.

THEOREM 3.9. Let $S$ be the spectrum of a Henselian discrete valuation ring $R$ with function field $K$ and with algebraically closed residue field. Let $X \rightarrow S$ be a smooth and surjective morphism, with $X$ a connected scheme. Let $G$ be an affine $K$-group scheme of finite type, and let $f: Y \rightarrow X_{\eta}$ be a $G$-torsor; then, there exist, possibly after extension of scalars, a connected scheme $X^{\prime}$ smooth and surjective over $S$, a model map $\lambda: X^{\prime} \rightarrow X$ and a $G_{0}$-torsor $f^{\prime}: Y_{0} \rightarrow X^{\prime}$ extending the given $G$-torsor $Y$ for some affine finite type and flat $S$-group scheme $G_{0}$. If, moreover, $G$ is finite, then $G_{0}$ is quasifinite, and there exists an open subscheme $X_{1} \subseteq X$ such that $X \backslash X_{1}$ has codimension $\geqslant 3$ in $X$ and such that $X^{\prime}$ can be obtained from $X_{1}$ after a finite number of Néron blowups.

The following corollary explains how to find models under the action of an étale group scheme when the given torsor is smooth. 
COROLlary 3.10. Let $S$ be the spectrum of a discrete valuation ring $R$ with function field $K$. Let $X \rightarrow S$ be a separated and faithfully flat morphism of finite type, with $X$ a regular and integral scheme of absolute dimension 2 endowed with a section $x \in X(S)$. Let $G$ be a finite étale $K$-group scheme, and let $f: Y \rightarrow X_{\eta}$ be a $G$-torsor pointed in $y \in Y_{x_{\eta}}(K)$; then, there exist an integral scheme $\widetilde{X}$ faithfully flat and of finite type over $S$, a model map $\lambda: \widetilde{X} \rightarrow X$ and a $\widetilde{G}$-torsor $\widetilde{f}: \widetilde{Y} \rightarrow \widetilde{X}$ extending the given $G$-torsor $Y$ for some quasi-finite and smooth $S$-group scheme $\widetilde{G}$. Moreover, $\widetilde{Y}$ comes with an $R$-point $\widetilde{y} \in \widetilde{Y}(R)$, whose restriction to $X_{\eta}$ is the given point $y$. Furthermore, $\widetilde{X}$ can be obtained from $X$ after a finite number of Néron blowups.

Proof. Let $X^{\prime}, G_{0}, Y_{0} \rightarrow X^{\prime}$ and $y_{0} \in Y_{0, x}(R)$ be as in Theorem 3.8. It is known that $G_{0}$ can be smoothened after a finite number of Néron blowups (see [1, Théorème 2.1.1]). We first Néron blow-up $G_{0}$ at some $H_{1} \leqslant$ $G_{0, s}$ (here, $G_{0, s}$ stands for the special fiber of $G_{0}$ ) in order to obtain $G_{0}^{H_{1}}$, then we Néron blow-up $G_{0}^{H_{1}}$ at $H_{2} \leqslant\left(G_{0}^{H_{1}}\right)_{s}$, and so on, obtaining a sequence $G_{n} \rightarrow G_{n-1} \rightarrow \cdots \rightarrow G_{0}$ of models of $G$ where $G_{n}$ is smooth. The group schemes $G_{i}$ that are flat models of finite type of the finite group $G$ are quasifinite. Therefore, clearly, if $G_{0}$ is smooth there is nothing to do. Otherwise, we first observe that we can always assume the special fiber $Y_{0, s} \rightarrow X_{s}^{\prime}$ of $Y_{0} \rightarrow X^{\prime}$ to be trivial; if not, it is sufficient to consider the Néron blowup $X^{\prime \prime}$ of $X^{\prime}$ at $x_{s}^{\prime}$ (the image in $X_{s}^{\prime}$ of $y_{0, s} \in Y_{0, s}$, which is closed in the special fiber) and to pull back $Y_{0} \rightarrow X^{\prime}$ over $X^{\prime \prime}$. Then, we can Néron blow it up at the trivial sub- $H_{1}$-torsor by means of Proposition 3.7, thus obtaining a $G_{0}^{H_{1}}$ torsor over some $X^{\prime \prime \prime}$, model of the given torsor. We go on like this, using the sequence of group schemes $H_{i}, i=1, \ldots, n$ introduced before. At each step, one gets a model $Y_{i} \rightarrow X_{i}$ of the original torsor of $Y \rightarrow X_{\eta}$ endowed by the universal property of Néron blowup, with sections $x_{i} \in X_{i}(R)$ and $y_{i} \in\left(Y_{i}\right)_{x_{i}}(R)$, whose restrictions to the generic fiber are $x_{\eta}$ and $y$.

A result similar to Corollary 3.10, mutatis mutandis, can be claimed under the assumptions of Theorem 3.9 too. Details are left to the reader.

Before proceeding with the proofs of Theorems 3.8 and 3.9, we need some preliminary results.

LEMMA 3.11. Let $S$ be a Dedekind scheme with function field $K$, and let $X \rightarrow S$ be a faithfully flat morphism of finite type with $X$ regular and integral. For any vector bundle $V$ on $X_{\eta}$, there exist an open subscheme $X_{1} \subseteq X$ containing $X_{\eta}$, where $X \backslash X_{1}$ has codimension $\geqslant 3$ in $X$, such that 
$X_{1}$ is faithfully flat and of finite type over $S$, and a vector bundle $W$ on $X_{1}$ such that $W_{\mid X_{\eta}} \simeq V$. If, moreover, $\operatorname{dim}(X)=2$, then we can choose $X_{1}=X$.

Proof. Let us denote by $j: X_{\eta} \rightarrow X$ the natural open immersion. First of all, we observe that there exists a coherent sheaf $\mathcal{F}$ on $X$ such that $j^{*}(\mathcal{F}) \simeq V$ (cf., for instance, [13, II, Example 5.15]). Then, $\mathcal{F}^{\vee \vee}$, that is the double dual of $\mathcal{F}$, is a coherent reflexive sheaf (see [12, Corollary 1.2]). That $j^{*}\left(\mathcal{F}^{\vee \vee}\right) \simeq V$ follows from the well-known fact that $j^{*}\left(\mathcal{F}^{\vee \vee}\right) \simeq j^{*}(\mathcal{F})^{\vee \vee} \simeq V$ (see, for instance, [12, proof of Proposition 1.8]). If $\operatorname{dim}(X)=2$, then, by $\left[12\right.$, Corollary 1.4], we set $W:=\mathcal{F}^{\vee}$, which is a vector bundle, and this is the last assertion. As for the higher-dimension case we know, again by [12, Corollary 1.4], that the subset $C$ of points where $\mathcal{F}^{\vee \vee}$ is not locally free is a closed subset of codimension $\geqslant 3$. We call $X_{1}$ the complementary open subset of $C$ in $X$ to which we give the induced scheme structure, and we thus know that $X_{1}$ contains $X_{\eta}$ and has nonempty intersection with $X_{s}$ for any closed point $s \in S$, whence we have the first assertion.

Let $T$ be any scheme. Following $[9,(11.6)]$, we associate to any locally free sheaf $V$ of rank $n$ over $T$ the sheaf $\mathcal{I}_{\text {som }_{\mathcal{O}_{T}}}\left(\mathcal{O}_{T}^{\oplus n}, V\right)$, which is a $\mathrm{GL}_{n, T}$-torsor $\mathcal{I}_{\text {som }_{\mathcal{O}_{T}}}\left(\mathcal{O}_{T}^{\oplus n}, V\right) \rightarrow T$, thus obtaining a bijective map between isomorphism classes of locally free sheaves of rank $n$ over $T$ and isomorphism classes of $\mathrm{GL}_{n, T}$-torsors over $T$. It is an exercise to prove that this construction base changes correctly (that is, if $i: T^{\prime} \rightarrow T$ is a morphism of schemes, then

$$
i^{*}\left(\mathcal{I}_{\operatorname{som}_{\mathcal{O}_{T}}}\left(\mathcal{O}_{T}^{\oplus n}, V\right)\right) \simeq \mathcal{I}_{\operatorname{som}_{\mathcal{O}_{T^{\prime}}}}\left(\mathcal{O}_{T^{\prime}}^{\oplus n}, i^{*}(V)\right)
$$

as $\mathrm{GL}_{n, T^{\prime}}$-torsors).

Proof of Theorem 3.8. We do the following construction. We take any closed immersion $\rho: G \hookrightarrow \mathrm{GL}_{n, K}$ for a suitable $n$ (by [25, Section 3.4]). The contracted product $Z:=Y \times{ }^{G} \mathrm{GL}_{n, K}$ via $\rho$ is a $\mathrm{GL}_{n, K}$-torsor; then, $Z \simeq \mathcal{I}_{\text {som }_{\mathcal{O}_{X_{\eta}}}}\left(\mathcal{O}_{X_{\eta}}^{n}, V\right)$ for a suitable vector bundle $V$ on $X_{\eta}$ (for instance, one can choose $\left.V:=f_{*}\left(\mathcal{O}_{Y}\right)\right)$ of rank $n$. Let $W$ be a vector bundle on $X$, as in Lemma 3.11, whose restriction to $X_{\eta}$ is isomorphic to $V$; let $Z^{\prime}:=\mathcal{I}_{\operatorname{som}_{\mathcal{O}_{X}}}\left(\mathcal{O}_{X}^{n}, W\right)$ be the corresponding $\mathrm{GL}_{n, S}$-torsor extending $Z$. Let us denote by $\bar{Y}$ and $\bar{G}$ the schematic closures of $Y$ in $Z^{\prime}$ and $G$ in $\mathrm{GL}_{n, S}$, 
respectively. We thus obtain the following diagrams:

(1)
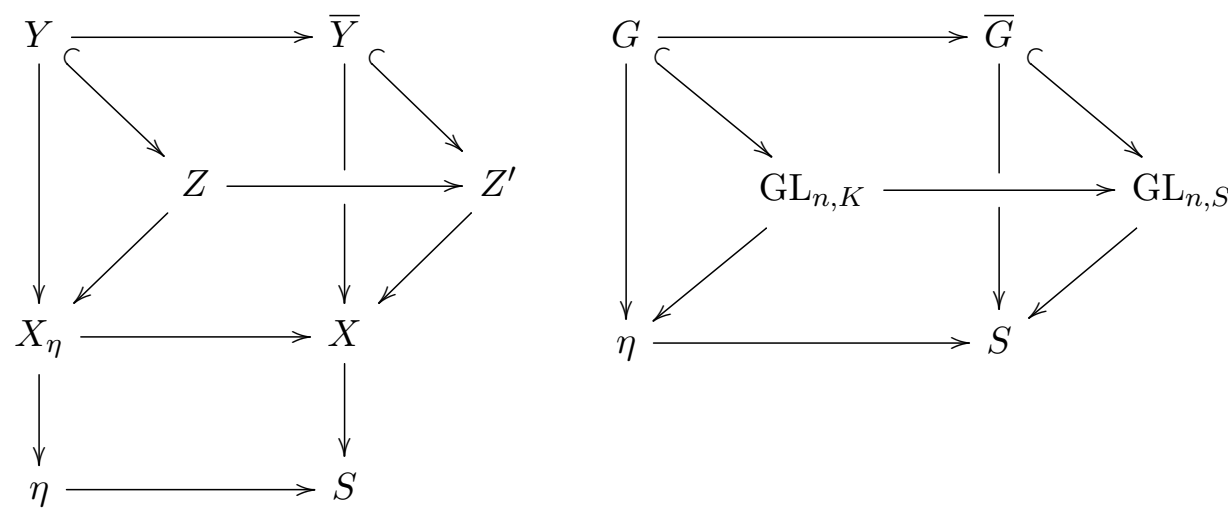

In general, neither $\bar{Y} \rightarrow X$ nor $\bar{Y} \rightarrow S$ will be faithfully flat. However, we can modify the embedding $G \hookrightarrow \mathrm{GL}_{n, K}$ in order to obtain at least the surjectivity of $\bar{Y} \rightarrow S$ (which will be thus faithfully flat). As we will see, this is sufficient to conclude. Let us now pull back the $\mathrm{GL}_{n, S^{-}}$-torsor $Z^{\prime} \rightarrow X$ over $x: \operatorname{Spec}(R) \rightarrow X$. We obtain a trivial torsor (see [16, III, Lemma 4.10]), whence we have the existence of a section $z^{\prime} \in Z^{\prime}{ }_{x}(S)$ whose generic fiber is $z_{\eta}^{\prime} \in Z_{x_{\eta}}(K)$. Let us now call $z \in Z_{x_{\eta}}(K)$ the image of $y$ through the closed embedding $i: Y \hookrightarrow Z$ constructed in diagram (1). In general, unless we are extremely lucky, it will not coincide with $z_{\eta}^{\prime}$, but, as they both live over $x_{\eta}$, there exists $g \in \mathrm{GL}_{n, K}(K)$ such that $z_{\eta}^{\prime}=z \cdot g$. Let us consider the isomorphism of $X_{\eta^{-}}$-schemes $\varphi_{g}: Z \rightarrow Z, z_{0} \mapsto z_{0} \cdot g$. The composition $\lambda:=\varphi_{g} \circ i: Y \hookrightarrow Z$ is a closed immersion sending $y$ to $z_{\eta}^{\prime}$, and it turns out to be a morphism of torsors, which is commuting with the actions of $G$ and $\mathrm{GL}_{n, K}$ if we consider the new embedding $\sigma: G \hookrightarrow \mathrm{GL}_{n, K}$ defined as $\sigma$ : $g_{0} \mapsto g^{-1} \rho\left(g_{0}\right) g$. If we now consider $\bar{Y}^{\prime}$ and $\bar{G}^{\prime}$, respectively, as the closure of $\lambda: Y \hookrightarrow Z$ in $Z^{\prime}$ and the closure of $\sigma: G \rightarrow \mathrm{GL}_{n, K}$ in $\mathrm{GL}_{n, R}$, then we observe that the natural morphism $u: \bar{Y}^{\prime} \times_{S} \bar{G}^{\prime} \rightarrow \bar{Y}^{\prime} \times{ }_{X} \bar{Y}^{\prime}$ is a closed immersion. This follows from the commutative diagram

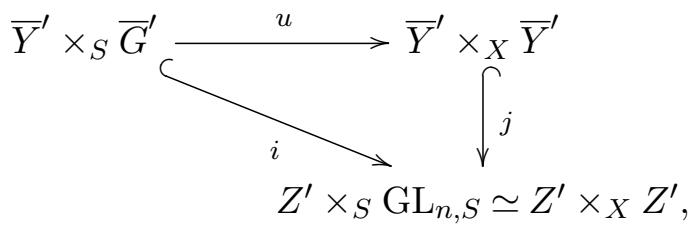


the fact that both $i: \bar{Y}^{\prime} \times_{S} \bar{G}^{\prime} \hookrightarrow Z^{\prime} \times_{S} \mathrm{GL}_{n, S}$ and $j: \bar{Y}^{\prime} \times_{X} \bar{Y}^{\prime} \rightarrow Z^{\prime} \times_{X} Z^{\prime}$ are closed immersions and [23, Lemma 28.3.1(3)]. Moreover, by construction, the closure of $y$ in $\bar{Y}^{\prime}$ has image $z^{\prime}$ in $Z^{\prime}$, so in particular $\bar{Y}^{\prime}$ is surjective (and thus faithfully flat) over $S$. According to Theorem 3.1, we set $X^{\prime}:=\bar{Y}^{\prime} / \bar{G}^{\prime}$, $Y_{0}:=\bar{Y}^{\prime}$ and $G_{0}:=\bar{G}^{\prime}$ in order to conclude. The fact that $X^{\prime} \rightarrow X$ can be obtained as a finite number of Néron blowups follows from [26, Theorem 1.4] and the fact that it is affine. Indeed, $\bar{Y} \rightarrow X$ is affine as the composition of the closed immersion $\bar{Y} \hookrightarrow Z^{\prime}$ and the $\mathrm{GL}_{n, R^{-}}$torsor $Z^{\prime} \rightarrow X$, and $\bar{Y} \rightarrow X^{\prime}=$ $\bar{Y} / \bar{G}^{\prime}$ is also affine.

Proof of Theorem 3.9. We repeat the first part of the proof of Theorem 3.8 in order to obtain a morphism of torsors $i: Y \hookrightarrow Z$, which is a closed immersion, from the given $G$ torsor to a $\mathrm{GL}_{n, K}$-torsor $Z:=$ $\mathcal{I}_{\text {som }_{\mathcal{O}_{X_{\eta}}}}\left(\mathcal{O}_{X_{\eta}}^{n}, V\right)$ for some vector bundle $V$ over $X_{\eta}$. Now, let $X_{1}$ be as in Lemma 3.11, and let $W$ be a vector bundle on $X_{1}$ whose restriction to $X_{\eta}$ is isomorphic to $V$; let $Z_{1}:=\mathcal{I}_{\operatorname{som}_{\mathcal{O}_{X_{1}}}}\left(\mathcal{O}_{X_{1}}^{n}, W\right)$ be the corresponding $\mathrm{GL}_{n, S^{-}}$ torsor extending $Z$. Let us denote by $\bar{Y}$ and $\bar{G}$ the schematic closures of $Y$ in $Z_{1}$ and $G$ in $\mathrm{GL}_{n, S}$, respectively. We thus obtain the following diagrams:
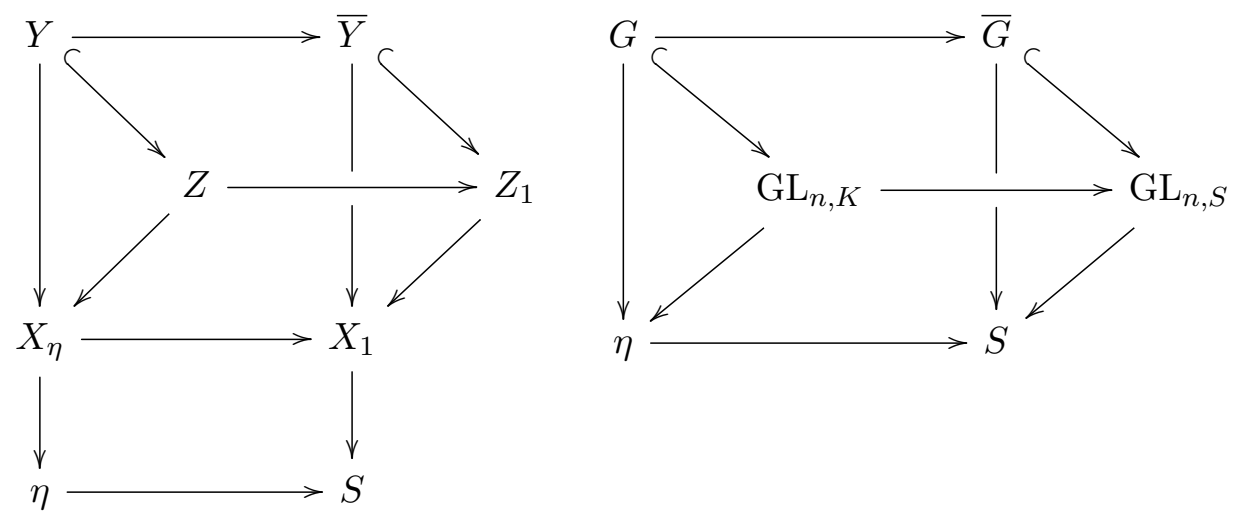

As $R$ is Henselian with algebraically closed residue field, and as $X_{1} \rightarrow S$ is smooth and surjective, there exists a section $x_{1} \in X_{1}(S)$. If necessary, after a finite extension of scalars $K \hookrightarrow K^{\prime}, Y_{K^{\prime}}$ is pointed over $x_{1, \eta}$; we can thus translate the problem to $R^{\prime}$, the Henselian ring obtained as the integral closure of $R$ in $K^{\prime}$. In order to ease notations, we assume $R=R^{\prime}$ from now on and we fix a point $y \in Y_{x_{1, \eta}}$. Arguing as in the proof of Theorem 3.8 , we can (and we actually do) assume that $i(y)=z_{1, \eta} \in Z_{x_{1, \eta}}(K)$, where 
$z_{1} \in Z_{1 x_{1}}(R)$ is an $R$-section of $Z_{1} \rightarrow S$, which always exists, as we have seen before; hence, $\bar{Y} \rightarrow S$ is faithfully flat. As in the proof of Theorem 3.8, $\bar{Y} \times_{S} \bar{G} \rightarrow \bar{Y} \times_{X_{1}} \bar{Y}$ is a closed immersion. Using Corollary 3.3, there exists a largest open $U$ of $\bar{Y}$, faithfully flat over $S$, such that $U \rightarrow U / \bar{G}$ is a $\bar{G}$ torsor extending the given one. If we set $X^{\prime}:=U / \bar{G}, G_{0}:=\bar{G}$ and $Y_{0}:=U$, then we obtain the desired result. If $G$ is finite, the fact that $U=\bar{Y}$ (we apply to the previous construction Theorem 3.1 instead of Corollary 3.3) implies the last assertion.

Acknowledgments. We would like to thank Hélène Esnault for very useful and helpful discussions and for pointing out a problem in a preliminary version. We would also like to thank Carlo Gasbarri for very interesting conversation on this subject. We are grateful to the referee for his/her very careful reading and his/her numerous remarks and suggestions which have improved our text.

\section{REFERENCES}

[1] S. Anantharaman, Schémas en groupes, espaces homogènes et espaces algébriques sur une base de dimension 1, Mém. Soc. Math. Fr. (N.S.) 33 (1973), 5-79.

[2] M. Antei, On the abelian fundamental group scheme of a family of varieties, Israel J. Math. 186 (2011), 427-446.

[3] M. Antei, Extension of finite solvable torsors over a curve, Manuscripta Math. 140(1) (2013), 179-194.

[4] M. Antei, M. Emsalem and C. Gasbarri, Sur l'existence du schéma en groupes fondamental, preprint, 2015, arXiv:1504.05082 [math.AG].

[5] S. Bosch, W. Lütkebohmert and M. Raynaud, Néron Models, Springer, Berlin, 1980.

[6] M. Demazure and P. Gabriel, Groupes algébriques, North-Holland, Amsterdam, 1970.

[7] N. G. Duong and P. H. Hai, Tannakian duality over Dedekind rings and applications, preprint, 2013, arXiv:1311.1134v2 [math.AG].

[8] C. Gasbarri, Heights of vector bundles and the fundamental group scheme of a curve, Duke Math. J. 117(2) (2003), 287-311.

[9] U. Görtz and T. Wedhorn, Algebraic Geometry I. Schemes. With Examples and Exercises, Advanced Lectures in Mathematics, Vieweg+Teubner, Wiesbaden.

[10] A. Grothendieck, Éléments de géomérie algébrique. IV. Étude locale des schémas et des morphismes de schémas. 2, Publ. Math. Inst. Hautes Études Sci. 24 (1965), 231 pp.

[11] A. Grothendieck, "Revêtements étales et groupe fondamental", in Fasc. II: Exposés 6, 8 à 11. Séminaire de géométrie algébrique, 1960/61, Troisiéme édition, corrigée Institut des Hautes Études Scientifiques, Paris, 1963, i+163 pp.

[12] R. Hartshorne, Stable reflexive sheaves, Math. Ann. 254 (1980), 121-176.

[13] R. Hartshorne, Algebraic Geometry, Graduate Texts in Mathematics, Springer, New York, Heidelberg, 1977.

[14] Q. Liu, Algebraic Geometry and Arithmetic Curves, Oxford Science Publications, Oxford, 2002. 
[15] V. B. Mehta and S. Subramanian, The fundamental group scheme of a smooth projective variety over a ring of Witt vectors, J. Ramanujan Math. Soc. 28A (2013), 341-351; special issue.

[16] J. S. Milne, Étale Cohomology, Princeton University Press, Princeton, NJ, 1980.

[17] J. S. Milne, Arithmetic Duality Theorems, Perspectives in Mathematics, 1, Academic Press, Boston, MA, 1986.

[18] M. V. Nori, The fundamental group-scheme, Proc. Indian Acad. Sci. Math. Sci. 91(2) (1982), $73-122$.

[19] M. V. Nori, The fundamental group-scheme of an Abelian variety, Math. Ann. 263 (1983), 263-266.

[20] M. Raynaud, Schémas en groupes de type $(p, \ldots, p)$, Bull. Soc. Math. France 102 (1974), 241-280.

[21] M. Raynaud, "Passage au quotient par une relation d'équivalence plate", in Proceedings of a Conference on Local Fields, Springer, Berlin, 1967, 78-85.

[22] M. Raynaud, p-groupes et réduction semi-stable des courbes, The Grothendieck Festschrift, Vol. III, Progr. Math., 88, Birkhäuser, Boston, MA, 1990, 179-197.

[23] Stack Project Authors, Stacks Project, version 94a58fd.

[24] D. Tossici, Effective models and extension of torsors over a d.v.r. of unequal characteristic, Int. Math. Res. Not. IMRN 2008 (2008), article ID rnn111, 68 pages.

[25] W. C. Waterhouse, Introduction to Affine Group Schemes, Graduate Texts in Mathematics, Springer, New York, Berlin, 1979.

[26] W. C. Waterhouse and B. Weisfeiler, One-dimensional affine group schemes, J. Algebra 66 (1980), 550-568.

Marco Antei

Laboratoire J. Dieudonné

Université de Nice Sophia Antipolis

Parc Valrose

Nice 06108

France

antei@unice.fr

Michel Emsalem

Laboratoire Paul Painlevé

U.F.R. de Mathématiques

Université des Sciences et des Technologies de Lille 1

Villeneuve d'Ascq 59655

France

emsalem@math.univ-lille1.fr 